\title{
Generation of Ultrasound Pulses in Water Using Granular Chains with a Finite Matching Layer
}

\author{
Sevan Harput, ${ }^{1}$ James McLaughlan, ${ }^{1,2}$ David M. J. Cowell, ${ }^{1}$ Pierre Gelat, ${ }^{3}$ Nader Saffari, ${ }^{3}$ Jia Yang, ${ }^{4}$ \\ Omololu Akanji, ${ }^{4}$ Peter J. Thomas, ${ }^{4}$ David A. Hutchins, ${ }^{4}$ and Steven Freear ${ }^{1, *}$ \\ ${ }^{1}$ Ultrasonics and Instrumentation Group, School of Electronic and Electrical Engineering, \\ University of Leeds, Leeds LS2 9JT, United Kingdom \\ ${ }^{2}$ Division of Biomedical Imaging, University of Leeds, Leeds LS2 9JT, United Kingdom \\ ${ }^{3}$ Department of Mechanical Engineering, University College London, Torrington Place, \\ London WCIE 7JE, United Kingdom \\ ${ }^{4}$ School of Engineering, University of Warwick, Coventry CV4 7AL, United Kingdom
}

(Received 26 June 2017; published 15 November 2017)

\begin{abstract}
Wave propagation in granular chains is subject to dispersive effects as well as nonlinear effects arising from the Hertzian contact law. This enables the formation of wideband pulses, which is a desirable feature in the context of diagnostic and therapeutic ultrasound applications. However, coupling of the ultrasonic energy from a chain of spheres into biological tissue is a big challenge. In order to improve the energy transfer efficiency into biological materials, a matching layer is required. A prototype device is designed to address this by using six aluminum spheres and a vitreous carbon matching layer. The matching layer and the precompression force are selected specifically to maximize the acoustic pressure in water and its bandwidth. The designed device generates a train of wideband ultrasonic pulses from a narrow-band input with a center frequency of $73 \mathrm{kHz}$. An analytical model is created to simulate the behavior of a matching layer as a flexible thin plate clamped from the edges. This model is then verified using free-field hydrophone measurements in water, which successfully predict the increased bandwidth by generation of harmonics. The shapes of the measured and predicted waveforms are compared by calculating the normalized cross-correlation, which shows $83 \%$ similarity between both. Since the generation of harmonics is of interest in this study, the total harmonic distortion (THD) and the -6-dB bandwidth of the signals are used to analyze signal fidelity between the hydrophone measurements and the model predictions. The acoustic signals in water have a root-mean-square THD of $73 \%$, and the model predicts a root-mean-square THD of 78\%. The -6-dB bandwidths of individual pulses measured by a hydrophone and predicted with the model are 280 and $252 \mathrm{kHz}$, respectively. At these high ultrasonic frequencies, it is an experimental demonstration of resonant chains operating in water with a matching layer.
\end{abstract}

DOI: 10.1103/PhysRevApplied.8.054032

\section{INTRODUCTION}

Wave propagation in homogeneous granular chains and interactions of spherical elastic objects have been well studied both theoretically and experimentally [1-5]. In such a system with Hertzian contact, a nonlinear forcedeformation relation exists as $F \propto \delta^{1.5}$. This nonlinear behavior can reshape the input of the system and generate wideband impulses similar to acoustic metamaterials and superlenses, which attract great attention due to their potential for improving spatial resolution beyond the diffraction limit [6]. It is also possible to use this nonlinear behavior in granular chains for acoustic switching and rectification via spectral bifurcation of certain frequencies into new broadband frequency components [7].

Multiple spherical chains can be used to form a nonlinear acoustic lens in a variety of applications such as biomedical

\footnotetext{
*Corresponding author. s.freear@leeds.ac.uk
}

imaging, nondestructive evaluation, underwater mapping, and shock absorbers [8-10]. Generation of wideband short-duration pulses is desirable both in diagnostic and therapeutic ultrasound. Wideband pulses improve the diagnostic image resolution and the functionality of other ultrasound modalities, such as harmonic imaging and multiple-excitation techniques [11-14]. In ultrasound therapy, short-duration monopolar ultrasonic pulses can create a spatially concentrated high-energy region, which minimizes the potential collateral damage in the surrounding healthy tissue [15].

Granular chains possess waveguidelike effects, such as turning corners and robust propagation under deflection [16]. The propagation characteristics, such as the propagation speed, can be tuned by changing the precompression force in the chain, particle material, or size. By using multiple granular chains, it is possible to focus and steer ultrasound waves $[8,9,17]$, which makes granular chains suitable for biomedical ultrasound applications by increasing the output pressure and the frequency. This study, 
therefore, focuses on maximizing the transferred energy from a chain of spheres into biological tissue and also the harmonic content, which increases the bandwidth of the generated ultrasound waves.

After Nesterenko's pioneering work in 1983 [1], a great number of models were developed to simulate wave propagation in infinite and finite chains $[2,4,18]$. However, predicting the resonance behavior in harmonically driven monoatomic granular chains, modeling the nonlinear and dispersive effects, and minimizing the overall structure are still big challenges $[5,19]$. In addition to this, the problem becomes more complicated once the chain is coupled to a finite material, where a matching layer is essential for biomedical applications. It is known that the wave interaction with boundaries depends on wall mechanical properties [20]. Coupling to a thin matching layer further increases the complexity of the existing problem, since the properties of the reflected and transmitted waves are significantly affected by both the material and its thickness [10].

$$
\begin{aligned}
m \ddot{x}_{1}= & \frac{4 \sqrt{R}}{3} \theta_{t-s}\left(\delta_{t-s}+x_{t}-x_{1}\right)^{\frac{3}{2}}-\frac{\sqrt{2 R}}{3} \theta_{s}\left(\delta_{s-s}+x_{1}-x_{2}\right)^{\frac{3}{2}} \\
& +\lambda\left(\dot{x}_{t}-\dot{x}_{1}\right) H\left(\delta_{t-s}+x_{t}-x_{1}\right) \\
& -\lambda\left(\dot{x}_{1}-\dot{x}_{2}\right) H\left(\delta_{s-s}+x_{1}-x_{2}\right), \\
m \ddot{x}_{i}= & \frac{\sqrt{2 R}}{3} \theta_{s}\left(\delta_{s-s}+x_{i-1}-x_{i}\right)^{\frac{3}{2}} \\
& -\frac{\sqrt{2 R}}{3} \theta_{s}\left(\delta_{s-s}+x_{i}-x_{i+1}\right)^{\frac{3}{2}} \\
& +\lambda\left(\dot{x}_{i-1}-\dot{x}_{i}\right) H\left(\delta_{s-s}+x_{i-1}-x_{i}\right) \\
& -\lambda\left(\dot{x}_{i}-\dot{x}_{i+1}\right) H\left(\delta_{s-s}+x_{i}-x_{i+1}\right), \\
m \ddot{x}_{n}= & \frac{\sqrt{2 R}}{3} \theta_{s}\left(\delta_{s-s}+x_{n-1}-x_{n}\right)^{\frac{3}{2}} \\
& -\frac{4 \sqrt{R}}{3} \theta_{m-s}\left(\delta_{m-s}+x_{n}-x_{m}\right)^{\frac{3}{2}} \\
& +\lambda\left(\dot{x}_{n-1}-\dot{x}_{n}\right) H\left(\delta_{s-s}+x_{n-1}-x_{n}\right) \\
& -\lambda_{\text {rest }}\left(\dot{x}_{n}-\dot{x}_{m}\right) H\left(\delta_{m-s}+x_{n}-x_{m}\right), \\
\frac{m_{m}}{4} \ddot{x}_{m}= & \frac{4 \sqrt{R}}{3} \theta_{m-s}\left(\delta_{m-s}+x_{n}-x_{m}\right)^{\frac{3}{2}} \\
& +\left(\lambda_{m}+\lambda_{\text {rest }}\right)\left(\dot{x}_{n}-\dot{x}_{m}\right) H\left(\delta_{m-s}+x_{n}-x_{m}\right) \\
& -K_{m} x_{m}-\lambda_{m} \dot{x}_{m} . \\
&
\end{aligned}
$$

A prototype device was developed based on this nonlinearity in a one-dimensional chain of spheres by Hutchins et al. [18,21-23]. The developed transducer is able to transform a narrow-band sinusoidal input force into a train of wideband impulses at ultrasonic frequencies. Donahue et al. [17] and Harput et al. [24,25] presented the generation of ultrasound waves in water by using a granular chain with a matching layer. However, neither of these studies analyzed the energy transfer from a chain of spheres into biological tissue in detail.

In this study, a matching layer is modeled as a flexible thin circular disk clamped from the edges and merged into an existing analytical model $[18,26]$ to identify suitable materials for biomedical applications. Different matching materials, such as glass, aluminum, acrylic, silicon rubber, and vitreous carbon, are analyzed with this model. The chosen matching material is attached to the aforementioned prototype device. The results achieved with this model are verified against hydrophone measurements.

\section{MATERIALS AND METHODS}

\section{A. Model with a matching layer}

An analytical model created by Hutchins et al. [18] and later analyzed in greater depth by Yang et al. [26] is used to simulate the wave propagation through the chain. The existing model that places an infinite wall behind the last sphere is modified to implement the effect of the matching layer at the end of the chain. The matching layer is modeled as a flexible thin plate clamped from the edges incorporated into the model as given in Eq. (4). Equations (1)-(3) describe the motion of the first sphere, middle spheres, and the last sphere for a granular chain consisting of $n$ spheres with a radius. The variable $m$ is the mass of a single sphere, $m_{m}$ is the mass of the matching layer, $x$ is the displacement, $\dot{x}$ is the velocity, and $\ddot{x}$ is the acceleration, where subscripts represent the following: 1 is the first sphere, $i$ is the $i$ th sphere, $n$ is the last sphere, $s$ is any sphere, $t$ is the ultrasonic horn, and $m$ is the matching layer. The damping of spheres $\lambda$ are considered only when the spheres are in contact by incorporating a Heaviside function, $H()$.

The effective Young's modulus $\theta$ of the spheres, ultrasonic horn, and matching layer are defined as

$\theta_{t}=\frac{E_{t}}{1-\nu_{t}^{2}}, \quad \theta_{s}=\frac{E_{s}}{1-\nu_{s}^{2}}, \quad \theta_{m}=\frac{E_{m}}{1-\nu_{m}^{2}}$,

with material properties $E$ corresponding to the Young's modulus, and $\nu$ corresponding to the Poisson ratio with the subscripts representing the same notation used above.

The effective Young's modulus associated with contact interactions between the first sphere and the ultrasonic horn is represented with $\theta_{t-s}$, and the same parameter between the last sphere and the matching layer is represented with $\theta_{m-s}$, which are defined as

$$
\frac{1}{\theta_{t-s}}=\frac{1}{\theta_{t}}+\frac{1}{\theta_{s}}, \quad \frac{1}{\theta_{m-s}}=\frac{1}{\theta_{m}}+\frac{1}{\theta_{s}} .
$$

The precompression force $F_{0}$ creates small elastic deformations, which result in an overlap of the hypothetical boundaries between the components of the system without 
any deformations. The overlap between spheres is denoted as $\delta_{s-s}$, the overlap between the first sphere and the ultrasonic horn is denoted as $\delta_{t-s}$, and the overlap between the last sphere and the matching layer is denoted as $\delta_{m-s}$. These parameters depend on the precompression force applied to the chain as

$$
\begin{aligned}
\delta_{t-s} & =\left(\frac{3 F_{0}}{4 \sqrt{R} \theta_{t-s}}\right)^{\frac{2}{3}}, \quad \delta_{s-s}=\left(\frac{3 F_{0}}{\sqrt{2 R} \theta_{s}}\right)^{\frac{2}{3}}, \\
\delta_{m-s} & =\left(\frac{3 F_{0}}{4 \sqrt{R} \theta_{m-s}}\right)^{\frac{2}{3}} .
\end{aligned}
$$

For the equation of motion of the matching layer given in Eq. (4), the first line of the equation describes the forces involved when the last sphere and the matching material are in contact, such as the Hertzian contact force and the deflection of the matching layer. The second line of the equation describes the free vibrations of the matching material as a thin plate clamped from the edges with an effective mass of $m_{m} / 4$ when the last sphere and matching layer are not in contact.

Deflection of a thin circular plate with clamped edges under a central load $F_{0}$ can be calculated according to the spring coefficient $K_{m}$ as

$$
\xi_{m}=\frac{F_{0}}{K_{m}}, \quad \text { for } K_{m}=\frac{4 \pi \theta_{m} h_{m}^{3}}{3 R_{m}^{2}},
$$

where $R_{m}$ and $h_{m}$ are the radius and the thickness of the matching layer, respectively.

Vibration of the matching plate is damped according to the damping coefficient associated with the matching layer $\lambda_{m}$ when not in contact with the last sphere. When the contact between the matching layer and the last sphere is regained, the damping due to restitutional motion $\lambda_{\text {rest }}$ becomes important, as given in Eq. (4). Yang et al. [10] suggested to use the inelasticity parameter $\lambda_{\text {rest }}$ to calculate the restitutional motion of the striker on a plate, which is determined by the aforementioned material properties, material density $\rho$, and geometry of the last sphere and the matching layer as

$\lambda_{\text {rest }}=\frac{\pi^{0.6}}{4 \sqrt{3}}\left(\frac{2 R}{h_{m}}\right)^{2}\left(\frac{\rho_{s}}{\rho_{m}}\right)^{0.6}\left(\dot{x}_{n} \sqrt{\frac{\rho_{m}}{\theta_{m}}}\right)^{0.2}\left(\frac{\theta s}{\theta s+\theta m}\right)^{0.4}$.

After deriving the equations of motion for the spheres and the matching layer, the mechanism of wave coupling into water is modeled as a superposition of waves propagating through the matching layer and vibrations of the matching layer as a thin plate. The propagated wave through the aluminum sphere into the matching layer and then from matching layer to water is attenuated due to an impedance mismatch between these layers as

$$
T_{m}=1-\left(\frac{Z_{m}-Z_{s}}{Z_{m}+Z_{s}}\right)^{2}, \quad T_{w}=1-\left(\frac{Z_{w}-Z_{m}}{Z_{w}+Z_{m}}\right)^{2},
$$

where $Z_{s}, Z_{m}$, and $Z_{w}$ are the acoustic impedance of the spheres, the matching layer, and water, respectively.

The final velocity at the surface of the matching layer $\dot{x}_{f}$ is calculated as the superposition of the last sphere's and matching layer's velocities as

$$
\dot{x}_{f}=\dot{x}_{n} \sqrt{T_{m} T_{w}} H\left(\delta_{m-s}+x_{n}-x_{m}\right)+\dot{x}_{m} .
$$

The first term in Eq. (11) is the velocity of the last sphere coupled to the matching layer and then water, while the last sphere is in contact with the matching layer. The transmitted energy is scaled by $T_{m}$ into the matching layer and $T_{w}$ into the water. The second term represents the velocity of the matching layer.

To reduce the complexity of the model, the following assumptions are made. The matching layer is modeled as a flexible thin circular disk clamped from the edges with an effective mass of $m_{m} / 4$. The Doppler effects due to the vibrations of the matching layer are ignored due to the large difference between the Doppler frequency and the output frequency of the system. The effect of nonlinear propagation of ultrasound waves in water is not incorporated into the model, since both the amplitude of the pressure wave and the ultrasonic frequency are low. The attenuation inside the matching material is ignored. The parameters used in the analytical model for different matching materials, spheres, and ultrasonic horn are assumed to be the generic material properties given in Table I.

\section{B. Experimental setup}

The experimental setup with the granular chain system and a membrane hydrophone is illustrated in Fig. 1. A granular chain containing six aluminum spheres with a diameter of $1 \mathrm{~mm}$ is placed into a holder. The holder is manufactured

\begin{tabular}{|c|c|c|c|c|}
\hline & $\begin{array}{l}\text { Density } \\
\left(\mathrm{kg} / \mathrm{m}^{3}\right)\end{array}$ & $\begin{array}{c}\text { Young's } \\
\text { modulus } \\
(\mathrm{GPa})\end{array}$ & $\begin{array}{c}\text { Poisson } \\
\text { ratio }\end{array}$ & $\begin{array}{l}\text { Acoustic } \\
\text { impedance } \\
\text { (MRayl) }\end{array}$ \\
\hline Aluminum & 2700 & 69 & 0.33 & 17.3 \\
\hline Stainless steel & 7800 & 200 & 0.30 & 45.7 \\
\hline Vitreous carbon & 1540 & 35 & 0.15 & 7.4 \\
\hline Acrylic & 1200 & 2.5 & 0.35 & 3.2 \\
\hline $\begin{array}{l}\text { Room-temperature- } \\
\text { vulcanizing silicone }\end{array}$ & 1100 & 0.1 & 0.49 & 1.4 \\
\hline Water & 1000 & & & 1.5 \\
\hline
\end{tabular}
from an R11 polymer by using the digital light processing technique with an EnvisionTEC Perfactory Mini Multi Lens 3D Printer (EnvisionTEC Inc., Dearborn, MI) to enhance the

TABLE I. Material parameters used in the analytical model. 


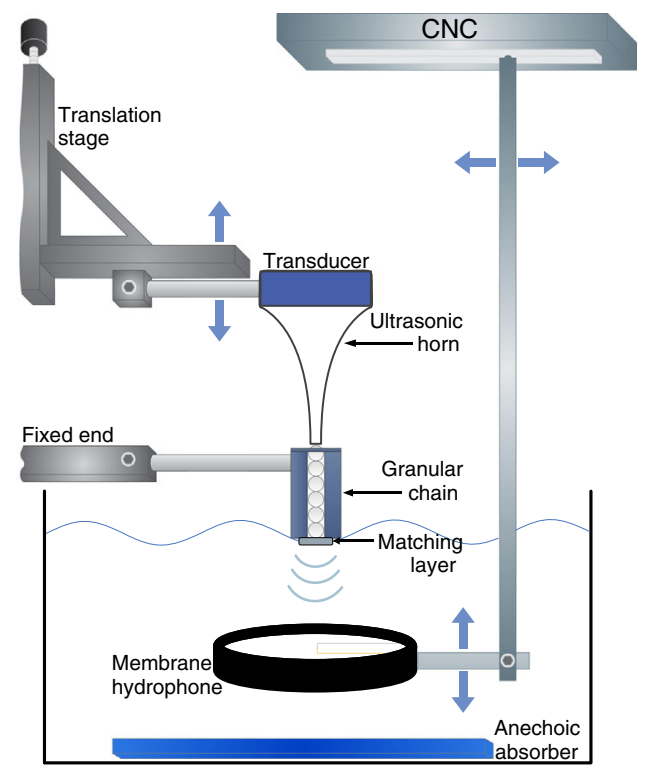

FIG. 1. Illustration of the experimental setup. Transducer, ultrasonic horn, granular chain assembly, and the matching layer together form a nonlinear system that can generate wideband impulses. A membrane hydrophone is used to measure the acoustic pressure waveform generated by this system. A computer numerical controller (CNC) is used to control the alignment of the hydrophone. The precompression force is adjusted with a high-precision micrometer translation stage. The granular chain is exaggerated for easier visualization.

solitary wave behavior as explained by Akanji et al. [23]. The chain of spheres and the ultrasonic horn are fixed on two separate assemblies, where a high-precision micrometer translation stage with a differential adjuster (Thorlabs, Newton, NJ) is used to adjust the static precompression force. The input into the granular chain is generated by an ultrasonic transducer attached to a stainless-steel ultrasonic horn to amplify the pressure waves generated by this transducer. A matching layer is placed on the other side of the chain to increase the coupling efficiency into water. A 0.5 -mm-thick vitreous carbon disk with a diameter of $12 \mathrm{~mm}$ is chosen as a matching layer with the given parameters in Table I. The system is partially submersed in degassed and deionized water for pressure measurements. The acoustic pressure generated by the granular chain is measured in water using a polyvinylidene fluoride differential membrane hydrophone (Precision Acoustics Ltd., Dorchester, UK) controlled by a CNC.

The transducer and the stainless-steel ultrasonic horn with a fundamental frequency of $73 \mathrm{kHz}$ is used to generate narrow-band signals as input into the chain of spheres. A 25-cycle sinusoidal tone burst with a central frequency of $73 \mathrm{kHz}$ is generated by a 33600A TrueForm waveform generator (Agilent Technologies Inc., Santa Clara, CA). This input waveform is amplified to $600 \mathrm{~V}$ p.p. by a $2200 \mathrm{~L}$ power amplifier (Electronics \& Innovation Ltd., Rochester, NY) before exciting the transducer. The displacement
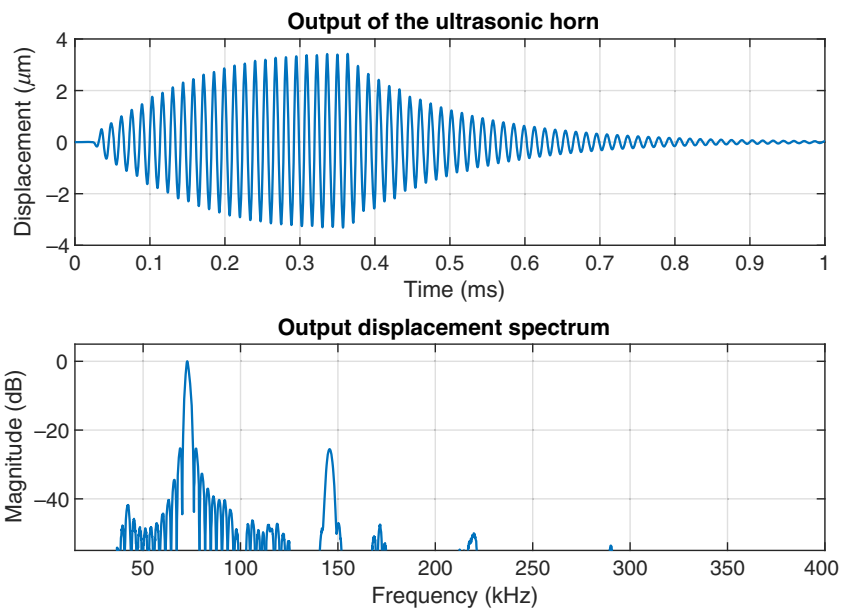

FIG. 2. (Top) Displacement profile measured at the tip of the ultrasonic horn with a laser vibrometer for a 25-cycle sine wave input with a center frequency of $73 \mathrm{kHz}$. (Bottom) Frequency spectrum of the measured displacement signal.

and the velocity profile at the tip of the ultrasonic horn is measured by a PSV-500 scanning vibrometer (Polytec $\mathrm{GmbH}$, Waldbronn, Germany). The measured displacement and velocity profiles by the laser vibrometer are used as input for the analytical model described above.

Figure 2 shows the input displacement profile of the granular chain measured at the tip of the ultrasonic horn and its spectrum. Because of the amplification effect of the horn, the input signal has a tapered window shape followed by a long ringing. This amplification is crucial since a single transducer cannot generate the necessary input displacement to trigger a nonlinear wave behavior in the chain. Although the output displacement profile looks different from a 25-cycle tone burst excitation, the spectra of the output signal show a narrow-band behavior with a $-6-\mathrm{dB}$ bandwidth of $3.5 \mathrm{kHz}$ and a second-harmonic level of $-26 \mathrm{~dB}$ as shown in Fig. 2 (bottom).

A positive push towards the hydrophone direction, as illustrated in Fig. 1, generates a compression wave, which is a negative pressure pulse in water. Therefore, all velocity plots presented here are inverted so as to enable direct comparison with the pressure plots.

\section{Precompression force}

The precompression force is estimated based on the resonance shift of the $73-\mathrm{kHz}$ ultrasonic horn under mechanical stress, similar to the principle used in atomic force microscopy [27]. A precompression force measurement setup is created (not shown in this paper) using calibrated masses and a Bode 100 vector network analyzer (Omicron Lab, Houston, TX) to detect the resonance peak. The ultrasonic horn is mechanically compressed using the calibrated masses, and its impedance is measured for precompression forces of $0-2 \mathrm{~N}$ with a step size of $0.25 \mathrm{~N}$. Therefore, the precompression force measurements have a 
precision of $0.25 \mathrm{~N}$ and a maximum error of $\pm 0.125 \mathrm{~N}$. After acquiring the impedance measurements, the ultrasonic horn is placed in the experimental setup shown in the Fig. 1, and the precompression force is adjusted with a micrometer translation stage to maximize the acoustic pressure amplitude in water, which corresponds to maximum velocity at the surface of the matching layer.

\section{RESULTS}

To demonstrate the effect of the matching layer, the response of the granular chain is calculated using the analytical model described earlier [18] for continuous sine wave excitation with an input displacement amplitude of $3.3 \mu \mathrm{m}$, input velocity of $1.5 \mathrm{~m} / \mathrm{s}, F_{0}=1.25 \mathrm{~N}$, and $\lambda=0.32$. Figure 3 shows the velocity of the last sphere for a six-sphere granular chain terminated with an infinite steel wall. A granular chain system placed between two immovable boundaries can accommodate periodic solitary waves for a given input force and precompression force, which is explained as in-phase nonlinear normal modes by Jayaprakash et al. [28].

After developing the model, different matching materials are analyzed to investigate the effect of the matching layer on the nonlinear wave generation in the granular chain. Stainless-steel, aluminum, vitreous carbon, acrylic, and silicon rubber matching layers are simulated with $0.5 \mathrm{~mm}$ thickness, where the wavelength for the slowest material corresponds to $13.8 \mathrm{~mm}$. Figure 4 shows the velocity at the surface of the matching layer, which can be considered as an approximate representation of pressure waves generated in water. The results show that soft matching layers such as rubber inhibit the generation of nonlinear periodic waves. This is intuitive, since the Young's modulus of the silicone rubber is $2-3$ orders of magnitude smaller than aluminum,
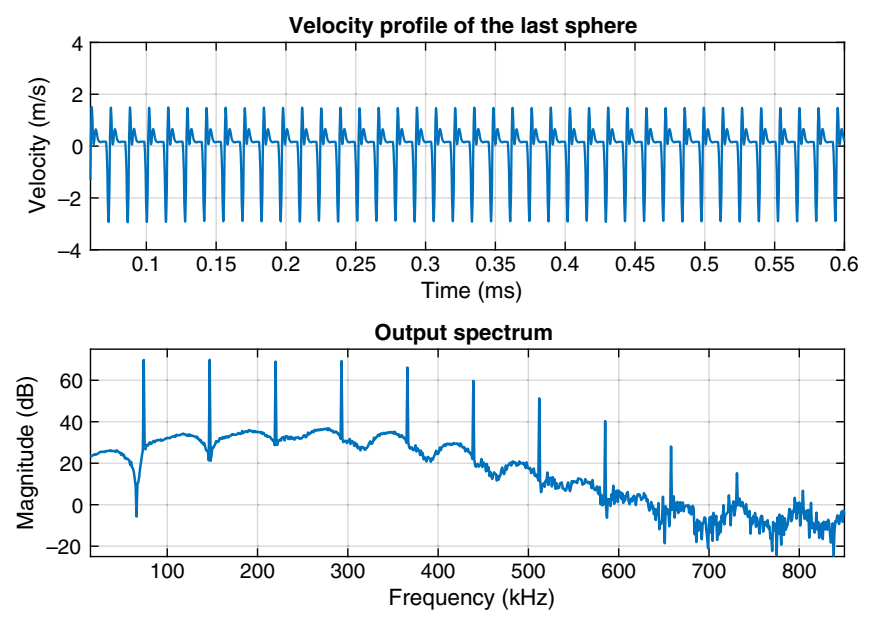

FIG. 3. (Top) Estimated velocity profile with the analytical model for a six-sphere chain against an immovable steel wall for precompression force of $1.25 \mathrm{~N}$. (Bottom) Corresponding frequency spectrum.
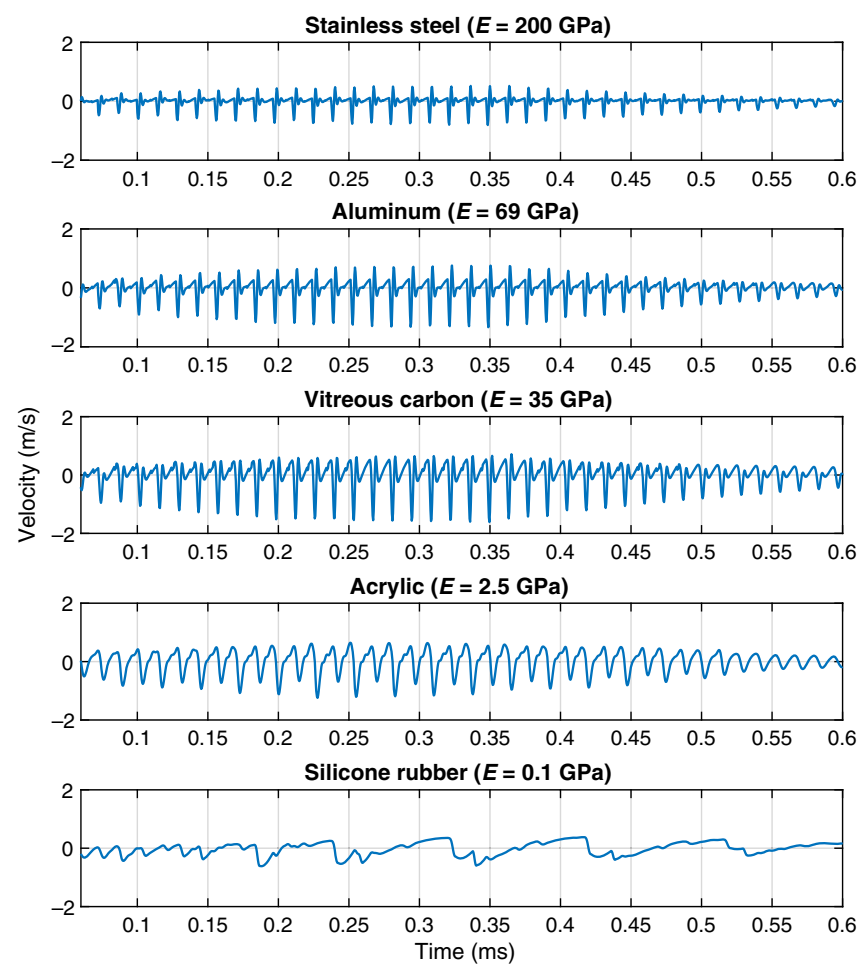

FIG. 4. Estimated velocity profile with the analytical model for a chain of six aluminum spheres terminated with different matching layers with a thickness of $0.5 \mathrm{~mm}$ and precompression force of $1.25 \mathrm{~N}$.

and the matching layer easily bends with the applied precompression force. In this case, the spheres may not easily lose contact with each other, which is necessary for the generation of nonlinear periodic solitary waves as shown in Fig. 3.

When a solitary wave reaches the end of the chain, the kinetic to potential energy conversion at the wall is a function of the Young's modulus, as demonstrated by Job et al. [20]. The ratio between the maximum force at the wall and the force observed by the sphere is proportional to $\left[E_{m} /\left(E_{m}+E_{s}\right)\right]^{0.4}$. This explains a large and small reduction in the amplitude of the periodic solitary waves, respectively, for the rubber and acrylic matching layers with small Young's modulus values. For larger Young's modulus values, Job et al. predicts an increased output force, which contradicts the findings of this study. The reason for this is due to the definition of the end location. Job et al. calculated the force at the end of the chain, which is the boundary between the last sphere and the wall. In this study, the proposed model predicts the velocity profile at the other side of the matching layer, which is in contact with water. For this case, the acoustic impedance, which is proportional to the square root of the Young's modulus and density, of the matching material becomes important. Therefore, when the behavior of the hard matching layer materials is compared, vitreous carbon achieves the largest peak velocity due to its acoustic impedance closer to the 
optimal value of $\sqrt{Z_{\text {aluminum }} Z_{\text {water }}}=5.1 \mathrm{MRayl}$. Aluminum, steel, and vitreous carbon matching layers all support the generation of nonlinear solitary waves, which can be seen in Fig. 4 as periodic nonlinear waves. However, for the aluminum and steel matching layers with a larger Young's modulus, the output velocity drops due to the increasing acoustic impedance mismatch.

After deciding on using vitreous carbon as a matching layer, the effect of the precompression force is simulated. The velocity profile and the corresponding frequency spectra at the surface of the vitreous carbon matching layer are estimated for different precompression forces. This study aims to maximize the output velocity profile or the pressure of the system for ultrasound applications, where the waveform shape and the amplitude of the generated waves are both important. Generation of a narrow negative solitary peak means a signal with high harmonic content, which is of interest for biomedical ultrasound applications. To demonstrate that it possible to maximize the output pressure by changing the input parameters, the system response for varying precompression force is plotted in Fig. 5 (top). For increasing precompression force up to $1 \mathrm{~N}$, the peak velocity increases, and the pulse width narrows. For the precompression force of $5 \mathrm{~N}$, the shape of the waveform changes and harmonics disappear. Figure 5 (bottom) shows the signal spectra for these velocity plots, where the spectrum of the waveform with $F_{0}=1 \mathrm{~N}$ has the highest magnitude at each harmonic, and, thus, it is the desirable signal.

For this reason, the relation between the precompression force and velocity profile must be analyzed first in order to
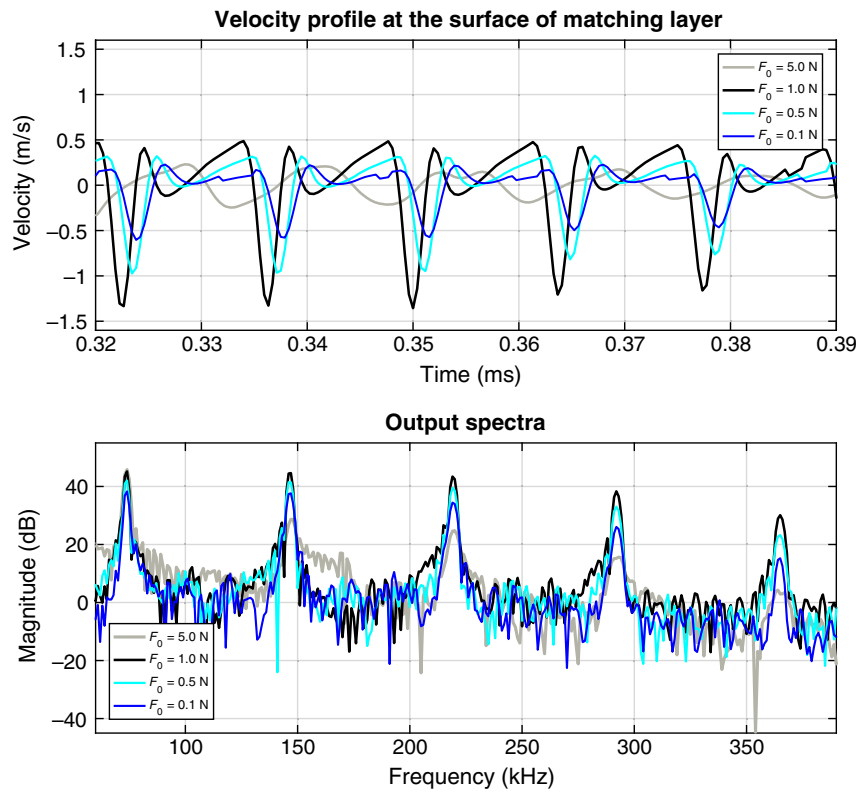

FIG. 5. (Top) Velocity profile estimated at the surface of the vitreous carbon matching layer for different precompression forces. (Bottom) Regarding frequency spectra.

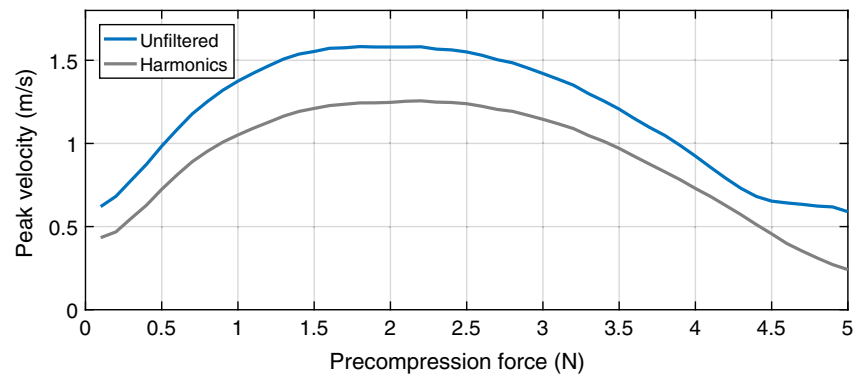

FIG. 6. Relation between the precompression force and maximum velocity profile at the surface of the matching layer that represents the acoustic pressure. The maximum harmonic content is calculated after filtering the fundamental signal at $73 \mathrm{kHz}$ with a high-pass filter.

increase the transferred ultrasound energy into biological tissue. Figure 6 shows that there exists an optimum precompression force for a given setup. Since the generation of higher-order harmonics increases the bandwidth of the ultrasound signal, a harmonic level is also included in the analysis, as shown in Fig. 6.

The maximum velocity amplitude is predicted by the analytical model to occur at a precompression force of $1.8 \mathrm{~N}$ (Fig. 6). It can be seen that there is very good qualitative agreement between the predictions of the analytical model and experiment. The experimental waveform seems more uniform in response across the harmonics, perhaps indicating a degree of additional damping in the experimental system, which is difficult to quantify but is likely to be due to damping of the horn caused by contact with the first sphere. Note that the maximum output signal is obtained using a precompression force of $1.25 \mathrm{~N}$. It is not possible to measure the excitation signal with a vibrometer while in contact; however, the loading effect changes the envelope shape of the excitation signal and also the peak displacement value, which directly reduces with the precompression force for the maximum velocity since $F_{\text {in }} / F_{0}$ is the scaling factor.

The granular chain setup is formed, and model parameters are estimated based on real hydrophone measurements. The precompression force applied on the chain is adjusted by using a differential adjuster translation stage, as shown in Fig 1. The experimental precompression force is estimated as $F_{0}=1.25 \mathrm{~N}$, while estimates for the damping coefficient yield $\lambda_{m}=1.05$ and $\lambda=0.32$ by matching the model with hydrophone measurements. These empirical parameters are used in this section for the coherency of the study.

Figures 7 and 8 show a comparison of the predicted waveform with the model and the ultrasound pressure waves measured with a hydrophone in water. Figure 7 (top) shows the measured and predicted waveforms for the whole duration. The measured signal exhibits a linear behavior for the first two to three cycles. Later on, the nonlinear behavior rises with increasing input force 

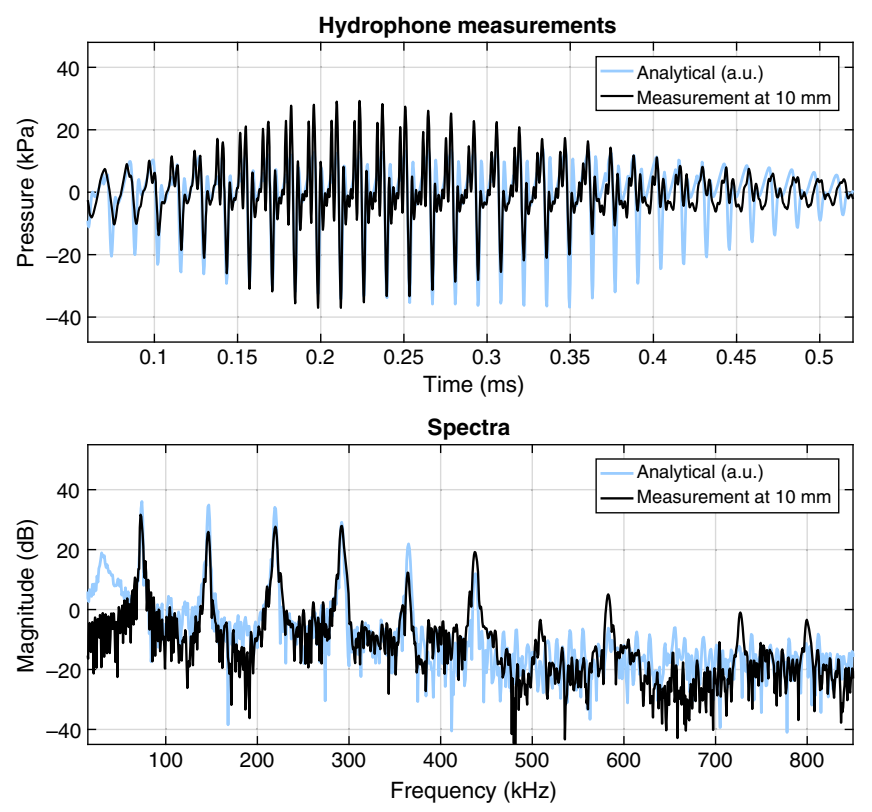

FIG. 7. (Top) Ultrasound pressure waveform measured in water and the velocity profile estimated at the surface of the vitreous carbon matching layer are plotted for comparison. (Bottom) Regarding frequency spectra.

(Fig. 2), which overlaps the predicted waveform by the model. After $t=0.35 \mathrm{~ms}$, the nonlinear behavior dies out faster than the model's prediction with several cycles of ringing. Figure 7 (bottom) shows spectra of the measured and predicted waveforms, where a rich harmonic content up to the 11th harmonic is observed for the measured signal.
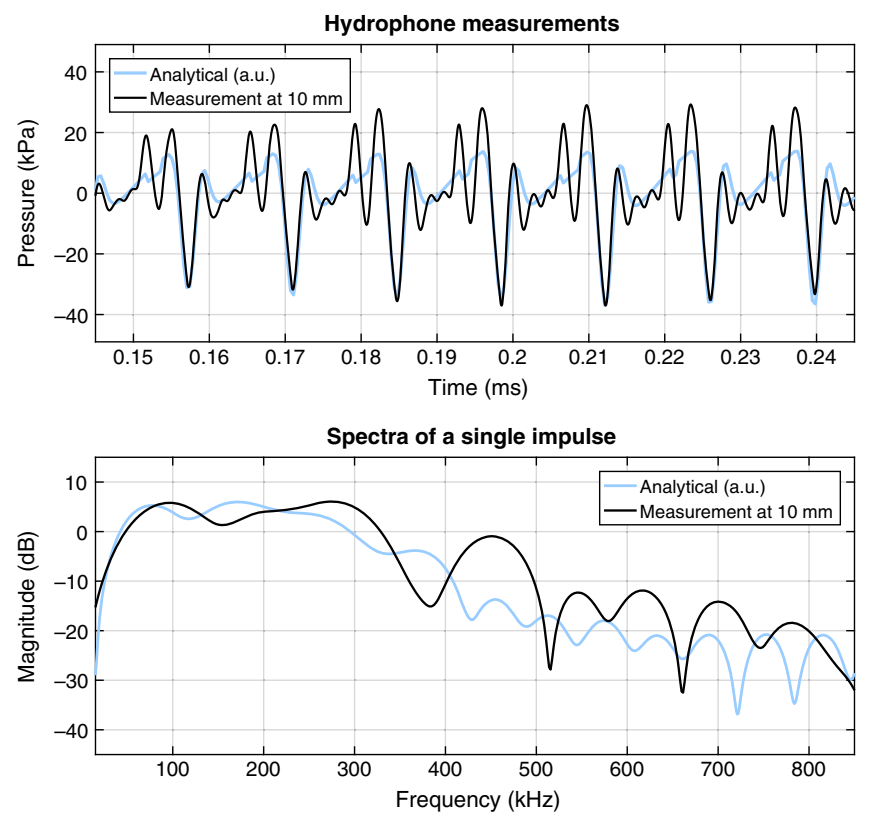

FIG. 8. (Top) Comparison of measured ultrasound wave and predicted velocity profile with the model. Figure 7 replotted with different time scale for clear visualization. (Bottom) Frequency spectra of single pulse located around $0.2 \mathrm{~ms}$.
For clear visualization of the signal envelope and individual pulse shapes, Fig. 7 is plotted again with a different time scale. To evaluate the correspondence of the predicted waveform to the measurements, three metrics are used: the normalized cross-correlation (NCC), -6-dB bandwidth of individual pulses as shown in Fig. 8 (bottom), and the root-mean-square total harmonic distortion (THD). The NCC shows similarities between the two signals in terms of shape and phase response. Although it is a good metric to evaluate the fidelity between two signals, the differences between the level of the harmonics and the signal bandwidth can be overlooked by NCC. Since the generation of harmonics is of interest in this study, the THD and the $-6-\mathrm{dB}$ bandwidth of the signals are also used to analyze signal fidelity between the hydrophone measurements and the model predictions.

The similarities between the two signals are calculated separately for all pulses between 0.11 and $0.38 \mathrm{~ms}$ in Fig. 7 (top). The average similarity between the measurements and the model is calculated as $83 \% \pm 3 \%$ by using NCC. The $-6-\mathrm{dB}$ bandwidth of the individual pulses is measured as $280 \mathrm{kHz}$, which is predicted with the model as $252 \mathrm{kHz}$. The root-mean-square THD is calculated to evaluate the harmonic energy generation up to the tenth harmonic, where THD values of $73 \%$ and $78 \%$ are measured with the hydrophone and predicted by the model, respectively.

Although the shape of the individual pulses matches with $83 \% \pm 3 \%$ correlation and the bandwidth with $10 \% \pm 4 \%$ error, the shape of the signal envelope is different as compared in Fig. 7. As outlined earlier, this is likely to be due predominantly by the loading effect on the ultrasonic horn when in contact with the spheres, which changes the measured displacement output of the horn shown in Fig. 2.

A comparison between the velocity profiles of the last (output) sphere and the matching layer is shown in Fig. 9. The amplitude at the surface of the matching layer facing the water is reduced as expected due to the impedance mismatch at either surface. The figure also highlights the

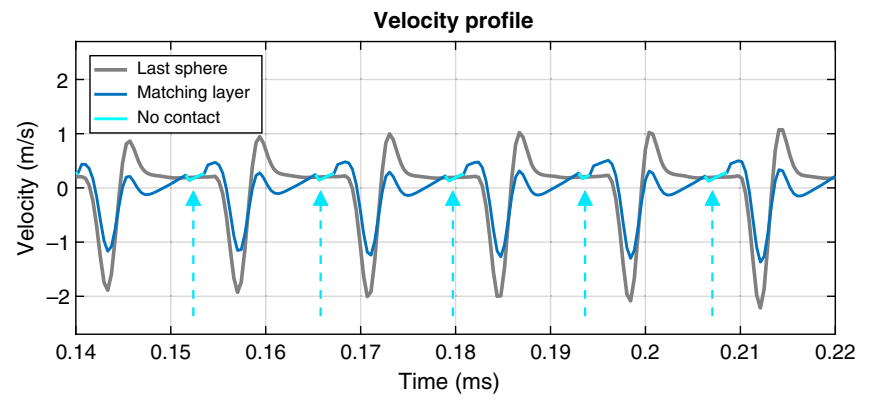

FIG. 9. Velocity profiles at the surface of the matching layer and the last sphere are plotted for comparison. Blue arrows point at the highlighted section of the waveform where the contact between the last sphere and the matching layer are lost. 
section of the waveform when the contact between the last sphere and the matching layer is lost.

\section{DISCUSSION}

In general, a reduction in the precompression force increases the nonlinearities in a granular chain, resulting in a larger energy transfer to higher frequencies [18]. However, after attaching a finite matching layer to the system, generation of wideband ultrasound waves in water cannot be maximized by reducing the precompression force. To maximize the amplitude of the ultrasound pressure waves, an optimum precompression force can be estimated based on the matching layer properties or an application-specific matching material can be selected. The model also shows that the selected material, its thickness, and, therefore, the mass of the matching layer affect the bandwidth of the output signal. A thin matching layer is ideal for coupling of higher-frequency signals; however, a thick layer increases the nonlinear behavior in the granular chain. Therefore, the traditional quarterwavelength matching approach is no longer suitable, since the final signal bandwidth is a function of the matching layer thickness. Although it is not performed in this study due to the lack of availability of different thickness vitreous carbon materials, the model can be used to predict an optimum matching layer thickness after incorporating the effect of attenuation and multiple reflections inside the material.

Another important difference of this study in comparison to others in the existing literature is the use of large precompression force $\left(F_{0}>1 \mathrm{~N}\right)$, which also brings certain advantages. The compression in the chain is set 3 orders of magnitude larger than the gravitational compression on a vertical chain, which eliminates the effect of gravity on the output of the system at any orientation and increases the mobility of the system. Note that it has been reported that the damping of the signals in a gravitationally compressed chain is different from that expected for a horizontal chain [29]. For a large precompression force, the characteristic of the wave propagation in the chain does not suddenly change due to small variations in precompression force and increases the tolerance of the system to external factors such as thermal effects. The large precompression force also increases the cutoff frequency of such chains, above which, solitary wave behavior is not expected. The cutoff frequency is usually estimated by assuming harmonic excitation of an infinite chain [1]. Such a cutoff frequency for a chain of aluminum spheres can be predicted in this way as $318 \mathrm{kHz}$ for $F_{0}=1.25 \mathrm{~N}$ and dropping to $209 \mathrm{kHz}$ for $F_{0}=0.1$. However, both in simulations and the experiment, harmonic generation can be seen at higher frequencies. This is a result of having a chain of finite length attached to an immovable (the ultrasonic horn) and a movable wall (the matching layer), where the cutoff frequency is not predictable due to the increased complexity of the system when a chain of finite length is used.

\section{CONCLUSIONS}

In this study, an analytical model is created to predict the motion of a finite material attached to a granular chain as a matching layer. An experimental setup is created to verify the estimated motion of the matching material with the new model. The setup consists of a one-dimensional chain of six aluminum spheres, a vitreous carbon matching layer, and an ultrasonic horn. The ultrasonic horn generates a narrowband input of a 25-cycle sinusoidal tone burst with a center frequency of $73 \mathrm{kHz}$ and a $-6-\mathrm{dB}$ bandwidth of $3.5 \mathrm{kHz}$. The output is measured in water as a train of wideband ultrasonic pulses with a $-6-\mathrm{dB}$ bandwidth of $280 \mathrm{kHz}$, which is predicted with the model as $252 \mathrm{kHz}$. The results presented in this paper indicate that such chainlike systems may be of interest to the biomedical ultrasound community, where a train of high-frequency impulses might have applications in imaging, microbubble dynamics, and highintensity therapy.

\section{ACKNOWLEDGMENTS}

The authors gratefully acknowledge funding from the Engineering and Physical Sciences Research Council (UK) via Grant No. EP/K029835/1, EP/K030159/1, and EP/ K032070/1.

[1] V.F. Nesterenko, Propagation of nonlinear compression pulses in granular media, J. Appl. Mech. Tech. Phys. 24, 733 (1984).

[2] C. Coste, E. Falcon, and S. Fauve, Solitary waves in a chain of beads under Hertz contact, Phys. Rev. E 56, 6104 (1997).

[3] F. Santibanez, R. Munoz, A. Caussarieu, S. Job, and F. Melo, Experimental evidence of solitary wave interaction in Hertzian chains, Phys. Rev. E 84, 026604 (2011).

[4] J. Lydon, K. R. Jayaprakash, D. Ngo, Y. Starosvetsky, A. F. Vakakis, and C. Daraio, Frequency bands of strongly nonlinear homogeneous granular systems, Phys. Rev. E 88, 012206 (2013).

[5] J. Lydon, G. Theocharis, and C. Daraio, Nonlinear resonances and energy transfer in finite granular chains, Phys. Rev. E 91, 023208 (2015).

[6] X. Zhang and Z. Liu, Superlenses to overcome the diffraction limit, Nat. Mater. 7, 435 (2008).

[7] N. Boechler, G. Theocharis, and C. Daraio, Bifurcationbased acoustic switching and rectification, Nat. Mater. 10, 665 (2011).

[8] K. Li, P. Rizzo, and X. Ni, Alternative designs of acoustic lenses based on nonlinear solitary waves, J. Appl. Mech. 81, 071011 (2014).

[9] A. Spadoni and C. Daraio, Generation and control of sound bullets with a nonlinear acoustic lens, Proc. Natl. Acad. Sci. U.S.A. 107, 7230 (2010). 
[10] J. Yang, D. Khatri, P. Anzel, and C. Daraio, Interaction of highly nonlinear solitary waves with thin plates, Int. J. Solids Struct. 49, 1463 (2012).

[11] F. Tranquart, N. Grenier, V. Eder, and L. Pourcelot, Clinical use of ultrasound tissue harmonic imaging, Ultrasound Med. Biol. 25, 889 (1999).

[12] Q. Ma, Y. Ma, X. Gong, and D. Zhang, Improvement of tissue harmonic imaging using the pulse-inversion technique, Ultrasound Med. Biol. 31, 889 (2005).

[13] A. Bouakaz and N. de Jong, Native tissue imaging at superharmonic frequencies, IEEE Trans. Ultrason. Ferroelectr. Freq. Control 50, 496 (2003).

[14] S. Harput, J. McLaughlan, D. M. J. Cowell, and S. Freear, Superharmonic imaging with chirp coded excitation: Filtering spectrally overlapped harmonics, IEEE Trans. Ultrason. Ferroelectr. Freq. Control 61, 1802 (2014).

[15] K.-W. Lin, T. L. Hall, R. J. McGough, Z. Xu, and C. A. Cain, Synthesis of monopolar ultrasound pulses for therapy: The frequency-compounding transducer, IEEE Trans. Ultrason. Ferroelectr. Freq. Control 61, 1123 (2014).

[16] L. Cai, J. Yang, P. Rizzo, X. Ni, and C. Daraio, Propagation of highly nonlinear solitary waves in a curved granular chain, Granular Matter 15, 357 (2013).

[17] C. M. Donahue, P. W. J. Anzel, L. Bonanomi, T. A. Keller, and C. Daraio, Experimental realization of a nonlinear acoustic lens with a tunable focus, Appl. Phys. Lett. 104, 014103 (2014).

[18] D. A. Hutchins, J. Yang, O. Akanji, P. J. Thomas, L. A. J. Davis, S. Freear, S. Harput, N. Saffari, and P. Gelat, Evolution of ultrasonic impulses in chains of spheres using resonant excitation, Europhys. Lett. 109, 54002 (2015).

[19] M. A. Porter, P. G. Kevrekidis, and C. Daraio, Granular crystals: Nonlinear dynamics meets materials engineering, Phys. Today 68, 44 (2015).

[20] S. Job, F. Melo, A. Sokolow, and S. Sen, How Hertzian Solitary Waves Interact with Boundaries in a $1 d$ Granular Medium, Phys. Rev. Lett. 94, 178002 (2005).

[21] D. A. Hutchins, J. Yang, O. Akanji, L. A. J. Davis, P. J. Thomas, S. Freear, S. Harput, N. Saffari, and P. Gelat,
The study of chain-like materials for use in biomedical ultrasound, in Proceedings of the IEEE International Ultrasonics Symposium (IUS), 2014, pp. 2607-2610, http://ieeexplore.ieee.org/document/6932096/.

[22] D. A. Hutchins, J. Yang, O. Akanji, P. J. Thomas, L. A. J. Davis, S. Freear, S. Harput, N. Saffari, and P. Gelat, Ultrasonic propagation in finite-length granular chains, Ultrasonics 69, 215 (2016).

[23] O. Akanji, J. Yang, D. A. Hutchins, P. J. Thomas, L. A. J. Davis, S. Harput, S. Freear, P. Gelat, and N. Saffari, The generation of impulses from narrow bandwidth signals using resonant spherical chains, IEEE Trans. Ultrason. Ferroelectr. Freq. Control 63, 1957 (2016).

[24] S. Harput, J. McLaughlan, S. Freear, P. Gelat, N. Saffari, J. Yang, O. Akanji, P. J. Thomas, and D. A. Hutchins, Nonlinear generation of harmonic content within high intensity ultrasound signals using granular chains, in Proceedings of the IEEE International Ultrasonics Symposium (IUS), 2015, http://ieeexplore.ieee.org/document/7329379/.

[25] S. Harput, S. Freear, P. Gelat, N. Saffari, J. Yang, O. Akanji, P. J. Thomas, and D. A. Hutchins, Coupling of wideband impulses generated by granular chains into liquids for biomedical applications, in Proceedings of the IEEE International Ultrasonics Symposium (IUS), 2016, http:// ieeexplore.ieee.org/document/7728481/.

[26] J. Yang, D. A. Hutchins, O. Akanji, P. J. Thomas, L. A. J. Davis, S. Harput, P. Gelat, S. Freear, and N. Saffari, An analysis of solitary wave impulses in granular chains using ultrasonic excitation, Phys. Rev. E 93, 063002 (2016).

[27] U. Rabe, K. Janser, and W. Arnold, Vibrations of free and surface-coupled atomic force microscope cantilevers: Theory and experiment, Rev. Sci. Instrum. 67, 3281 (1996).

[28] K. R. Jayaprakash, Y. Starosvetsky, A. F. Vakakis, M. Peeters, and G. Kerschen, Nonlinear normal modes and band zones in granular chains with no pre-compression, Nonlinear Dyn. 63, 359 (2011).

[29] J. Hong, H. Kim, and J.-P. Hwang, Characterization of soliton damping in the granular chain under gravity, Phys. Rev. E 61, 964 (2000). 